\title{
Pathogenesis of intestinal Pseudomonas aeruginosa infection in patients with cancer
}

\author{
Panayiota Markou and Yiorgos Apidianakis* \\ Department of Biological Sciences, University of Cyprus, Nicosia, Cyprus \\ ${ }^{*}$ Correspondence: apidiana@ucy.ac.cy \\ Edited by: \\ Dominique Ferrandon, Centre National de la Recherche Scientifique, France \\ Reviewed by: \\ John C. Alverdy, University of Chicago, USA
}

Keywords: inflammation, tumour, Drosophila, human, gut, epithelial damage, regeneration

In 1882, Robert Koch suggested four postulates that establish causation between an infectious agent and a particular disease: (1) the infectious organism must be found in abundance in all diseased, but not in healthy, organisms, (2) the infectious organism must be isolated from the diseased host and grown in culture, (3) the disease must be reproduced when the cultured organism is introduced into a healthy organism and (4) the same organism must be reisolated from the experimentally diseased host (Tabrah, 2011; Breitschwerdt et al., 2013). In Figure 1 we suggest an adaptation to the original postulates of Koch to be used as a framework to assess the causation between intestinal Pseudomonas aeruginosa and intestinal disease in patients with cancer. In the following sections we describe the prevalence of $P$. aeruginosa in cancer and the immunosuppressive and stress-inducing conditions of cancer that facilitate the growth, dissemination and virulence of intestinal $P$ aeruginosa. In addition, we describe work showing that $P$. aeruginosa promotes intestinal epithelium cancer-related phenotypes when introduced in tumor prone model hosts.

\section{CANCER AND OTHER IMMUNOSUPPRESSIVE CONDITIONS PROMOTE THE PREVALENCE OF $P$. aeruginosa}

Bacteremia is a major cause of lifethreatening complications in patients with cancer, especially those who receive anticancer chemotherapy. Cancer patients are more vulnerable to invasive infection, due to ulcerative lesions in mucosal surfaces and immune suppression secondary to chemotherapy (Safdar and
Armstrong, 2001). Many studies associate bloodstream infections in cancer patients with Gram-negative bacteria (Oliveira et al., 2007; Bos et al., 2013; Montassier et al., 2013).

$P$. aeruginosa is a Gram-negative opportunistic bacterium that causes various infections. Common communityacquired infections with $P$. aeruginosa are skin and soft tissue infections, ulcerative keratitis and otitis externa, while hospitalacquired infections include bloodstream infections, pneumonias and urinary tract infections (Driscoll et al., 2007). Infections may be associated with a high rate of morbidity and mortality in immunocompromised hosts, such as those suffering from chemotherapy-induced neutropenia, patients with cystic fibrosis or severe burns and individuals who receive intensive care (Driscoll et al., 2007; Kerr and Snelling, 2009; Worth and Slavin, 2009; Stuart et al., 2010; Rafla and Tredget, 2011).

$P$. aeruginosa intestinal carriage increases from $\sim 3 \%$ in normal people to $\sim 20 \%$ in hospitalized patients (Stoodley and Thom, 1970). In a case-control study the intestinal colonization by $P$. aeruginosa in cancer patients was $10 \%$ before and $31 \%$ after hospitalization (Andremont et al., 1989). Studies conducted in oncologyhematology units, found an overall intestinal carriage of $P$. aeruginosa between 11.7 and 37\% (Thuong et al., 2003). In another case-control study $P$. aeruginosa intestinal colonization was identified in $17 \%$ of controls and $60 \%$ of blood cancer patients (Vuotto et al., 2013). These epidemiology data suggest that intestinal colonization by $P$. aeruginosa is prominent among hospitalized cancer patients
(Andremont et al., 1989; Vuotto et al., 2013).

The intestinal carriage of $P$. aeruginosa is likely a consequence of the opportunistic nature of this species. Most $P$. aeruginosa infections appear secondary to a breach in host defences. In addition to compromised host immunity, intestinal microbiota play a major role in intestinal defence to infection (Levison, 1973). Thus systemic exposure to antibiotics, which alters intestinal microbiota by reducing the abundance of certain microbes creates the opportunity for intestinal growth of $P$. aeruginosa and other pathogenic bacteria (Hentges et al., 1985).

\section{INTESTINAL $P$. aeruginosa AS A SOURCE OF SYSTEMIC AND REMOTE INFECTIONS}

The translocation of endogenous intestinal $P$. aeruginosa extraluminally is an important pathogenic phenomenon and a cause of systemic infections, especially in neutropenic patients with hematological malignancies (Okuda et al., 2010). During the translocation process, bacteria and their products cross the intestinal barrier by traveling between or through the cells of the intestinal epithelium, causing infection and massive inflammation (Alexander et al., 1990; Papoff et al., 2012). Lung infections caused by $P$. aeruginosa are frequent in patients and can occur by direct contamination of the lungs by gastrointestinal flora or through hematogenous spread from the intestine to the lungs. Sepsis and mortality in immunocompromised patients are the results of the presence of highly virulent strains of $P$. aeruginosa within the intestinal tract and the pathogen's ability to adhere to the 


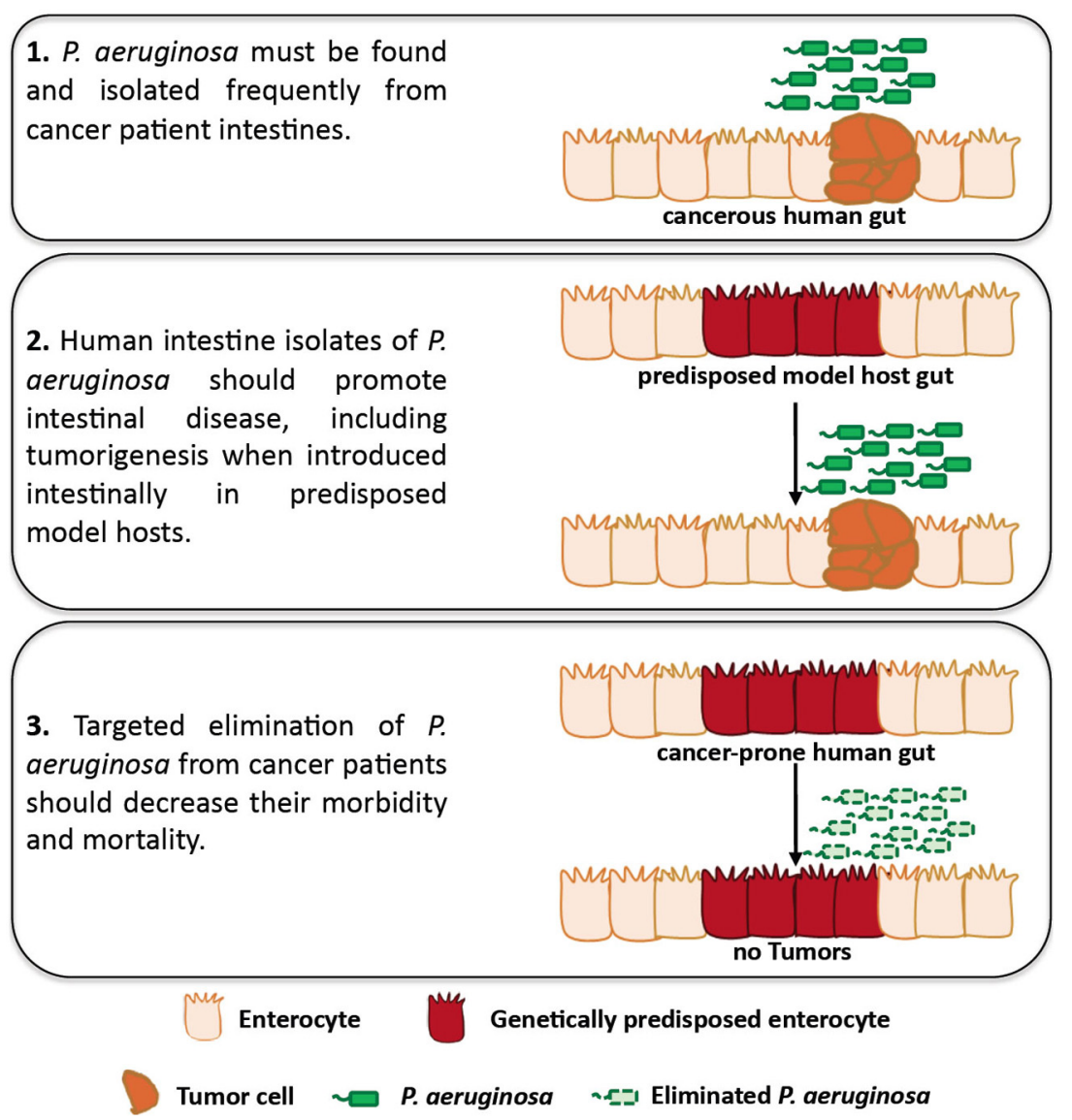

FIGURE 1 | An adaptation of Koch's postulates assessing causation between intestinal $P$. aeruginosa and disease in patients with cancer. Studies using Drosophila and mammalian hosts may assess the role of $P$. aeruginosa in facilitating intestinal disease, including intestinal $P$. aeruginosa growth, dissemination, virulence and tumorigenesis, in predisposed hosts. In addition, clinical studies can be designed to assess the presence of $P$. aeruginosa in cancer patients and its role in intestinal disease, including tumorigenesis for which clinical data are lacking. intestinal epithelial barrier (Marshall et al., 1993; Alverdy et al., 2000; Osmon et al., 2004; Zaborina et al., 2006; Okuda et al., 2010).

$P$. aeruginosa uses different virulence factors that can damage epithelial cells, such as enzymes (proteases and elastases), toxins, adhesins, flagella and protein secretion systems (Sundin et al., 2004). The T3SS enables the injection of at least four effector proteins (ExoS, ExoT, ExoU, and ExoY) into the host cell. ExoS injected into the host epithelial cell migrates to the membrane where it binds to the mammalian factor FXYD3, expressed specifically in the colon and stomach (Okuda et al., 2010). Thus, ExoS may assist the penetration of $P$. aeruginosa through the intestinal epithelial barrier, impairing the defence function of tight junctions against bacterial penetration (Okuda et al., 2010). Moreover, gut inflammation and apoptosis-which can be initiated by the Pseudomonas quinolone signal (PQS)lead to tight junction disruption and an increase of epithelial barrier permeability (Alverdy et al., 2005; An, 2008). Similarly, P. aeruginosa lectin PA-I, which is associated with adhesion to epithelial cell layer, is produced after intestinal ischemia and secreted into the intestinal lumen, causing tight junction interruption, epithelial barrier dysfunction and increase of its permeability (Seal et al., 2011).

\section{INTESTINAL $\boldsymbol{P}$. aeruginosa EXHIBITS ENHANCED VIRULENCE UPON STRESS, SURGERY, TRAUMA, AND MAYBE CANCER}

Cohort studies show that infections are more frequent, severe and lethal among surgical patients (Craven et al., 1988;
Sax et al., 2011). Surgical injury can shift the dynamics of the host-pathogen interaction leading to phenotype transformation or phase variation that develops as microbes adapt and respond to novel environments, causing morbidity and mortality (Babrowski et al., 2013). P. aeruginosa escalates its virulence and promotes systemic inflammation during various conditions of host stress (Seal et al., 2011). In patients colonized by $P$. aeruginosa, the prolonged surgical injury releases stress-related host factors that can trigger the otherwise dormant colonizers, making them invasive and lethal (Babrowski et al., 2013). Defence mechanisms such as degradative proteases and lipases, exopolysaccharide capsule and outer membrane-derived vesicles (OMVs), which serve as a secretion mechanism for virulence factors, 
help the pathogen to survive in the host environment (Macdonald and Kuehn, 2013). OMVs are induced in response to physiological stressors and secreted during infection serving multiple roles in bacterial pathogenesis (Macdonald and Kuehn, 2013). In surgically stressed hosts interferon-gamma, endogenous opioids and the hypoxic end-products adenosine and inosine are released into the intestinal lumen where they bind bacteria and activate the expression of PA-I lectin and other virulence factors of $P$. aeruginosa. The PA-I lectin alters the tight junction permeability of the intestinal epithelium to exotoxin $\mathrm{A}$, leading to lethal gut derived sepsis (Long et al., 2008). Moreover, local intestinal depletion of extracellular phosphate (hypophosphatemia), which occurs after surgical injury, can activate virulent pathways due to bacterial sensing of low phosphate, shifting the phenotype of $P$. aeruginosa to that of a lethal strain (Long et al., 2008). Because interferon-gamma, opioids and hypoxia are part of the host response and the therapeutic regiments administered to cancer patients (Dunn et al., 2005; Vaupel and Mayer, 2007), the conditions that accompany cancer may also provide the signals for $P$. aeruginosa virulence induction.

\section{CAN $P$. aeruginosa SIMILARLY TO OTHER GASTROINTESTINAL BACTERIA FACILITATE CANCER?}

Bacteria may initiate oncogenesis because they can induce inflammation and produce cell damaging toxins that facilitate tumorigenesis (Collins et al., 2011; Tjalsma et al., 2012). The characteristic single polar flagella and type 4 pili of $P$. aeruginosa function as initiators of inflammation and adhesins, respectively (Gellatly and Hancock, 2013). P. aeruginosa induces Toll-like receptors to activate cytokines, chemokines and COX-2 and recruit cells of the innate and adaptive immune system (Hussain et al., 2003; Holt et al., 2008; de Lima et al., 2012). Epithelial adherence is a property of various bacteria associated with gastrointestinal disease and cancer, such as Bacteroides fragilis, Streptococcus bovis, Escherichia coli and Helicobacter pylori (Toprak et al., 2006; Selgrad et al., 2008; Abdulamir et al., 2011; Arthur et al., 2012). Cell wall antigens of $S$. bovis induce overexpression of COX-2 and
NF-кB in vitro, which promote cellular proliferation and angiogenesis (Tafe and Ruoff, 2007; Abdulamir et al., 2009).

E-cadherin, a cell adhesion molecule serves as an antagonist of invasion and metastasis and is found mutated in human carcinomas (Cavallaro and Christofori, 2004; Berx and van Roy, 2009). B. fragilis secreted factor BFT cleaves E-cadherin, which is usually bound inside the plasma membrane to $\beta$-catenin. The cleavage releases catenin in the cytosol leading to the transcription of the oncogene $c-m y c$ (Hardy et al., 2000). Similarly, P. aeruginosa secreted factor LasI can disrupt adherens junctions and reduce the expression and distribution of E-cadherin and $\beta$-catenin in the cell membrane, resulting in changes in cell junction associations and enhanced paracellular permeability (Vikström et al., 2009).

Interestingly, intestinal innate immune responses and stem cells may drive tumor initiation, maintenance and metastasis (Schwitalla et al., 2013). Cancer development is assisted by apoptotic programmed cell death in the tumor microenvironment (Evan and Littlewood, 1998; Lowe et al., 2004; Adams and Cory, 2007) and $P$. aeruginosa uses many virulence factors that induce epithelial cell apoptosis. Intestinal infection with $P$. aeruginosa in Drosophila activates the c-Jun $\mathrm{N}$-terminal kinase (JNK) pathway, which causes apoptosis of enterocytes and leads to proliferation of intestinal stem cells (Apidianakis et al., 2009). Importantly, genetic predisposition via an oncogenic form of Ras1/K-Ras oncogene, can synergize with inflammatory signals to induce stem cell-originating tumors characterized by alterations in cell polarity and tissue architecture. Moreover, sustained intestinal infection with $P$. aeruginosa in Drosophila induces the Imd/NF- $\mathrm{BB}$ pathway, which synergizes with the oncogene Ras $1^{V 12}$ to activate the JNK pathway. This leads to invasion and dissemination of oncogenic hindgut cells to distant sites (Bangi et al., 2012; Christofi and Apidianakis, 2013).

\section{CONCLUSIONS}

$P$. aeruginosa is a common colonizer of the human intestine upon hospitalization, immunosuppression, antibiotic treatment, surgery, severe trauma and other conditions that cancer patients may face. Not only is $P$. aeruginosa carriage increased in the aforementioned conditions, but also bacteria become more virulent and damaging to the intestinal epithelium upon surgery, injury, and severe stress. Moreover, human isolates of $P$. aeruginosa can induce intestinal pathology and cancer-related epithelial phenotypes in genetically predisposed model hosts. Thus, $P$. aeruginosa appears to have the opportunity and the ability to promote intestinal disease in predisposed hosts, although further proof on the ability of this bacterium to promote tumorigenesis in mammalian models of infection is needed. The lack of epidemiological data linking $P$. aeruginosa to intestinal disease and potentially tumorigenesis in cancer patients may reflect the lack of clinical studies assessing bacterial growth and virulence in relation to cancer recurrence. Because the titter, distribution and virulence of $P$. aeruginosa in the intestine may be very dynamic (Tjalsma et al., 2012), future studies should be designed to repeatedly assess intestinal $P$. aeruginosa abundance and virulence in cancer patients versus healthy individuals. Clinical samples can be assessed for the presence of $P$. aeruginosa via classical microbiology, and next-generation sequencing may offer the chance to assess $P$. aeruginosa transcriptome during infection. Importantly, definite proof of causation of $P$. aeruginosa in morbidity and mortality of cancer patients can only be achieved if targeted elimination of $P$. aeruginosa from these patients improves the outcome of their disease. In Figure 1 we illustrate a roadmap to specifically assess the role of $P$. aeruginosa in intestinal disease and tumorigenesis. It is conceivable that similar principles can be used to assess causality between intestinal disease and many other opportunistic pathogens harbored by the human gut.

\section{REFERENCES}

Abdulamir, A. S., Hafidh, R. R., and Abu Bakar, F. (2011). The association of streptococcus bovis/gallolyticus with colorectal tumors: the nature and the underlying mechanisms of its etiological role. J. Exp. Clin. Cancer Res. 30, 11. doi: 10.1186/1756-9966-30-11

Abdulamir, A. S., Hafidh, R. R., Mahdi, L. K., Al-jeboori, T., and Abubaker, F. (2009). Investigation into the controversial association of streptococcus gallolyticus with colorectal 
cancer and adenoma. BMC Cancer 9:403. doi: 10.1186/1471-2407-9-403

Adams, J. M., and Cory, S. (2007). The bcl2 apoptotic switch in cancer development and therapy. Oncogene 26, 1324-1337. doi: 10.1038/sj.onc. 1210220

Alexander, J. W., Boyce, S. T., Babcock, G. F., Gianotti, L., Peck, M. D., Dunn, D. L., et al. (1990). The process of microbial translocation. Ann. Surg. 212, 496-510. discussion: 511-512. doi: 10.1097/00000658-199010000-00012

Alverdy, J., Holbrook, C., Rocha, F., Seiden, L., Wu, R. L., Musch, M., et al. (2000). Gutderived sepsis occurs when the right pathogen with the right virulence genes meets the right host: evidence for in vivo virulence expression in Pseudomonas aeruginosa. Ann. Surg. 232, 480-489. doi: 10.1097/00000658-200010000-00003

Alverdy, J., Zaborina, O., and Wu, L. (2005). The impact of stress and nutrition on bacterial-host interactions at the intestinal epithelial surface. Curr. Opin. Clin. Nutr. Metab. Care 8, 205-209. doi: 10.1097/00075197-200503000-00016

An, G. (2008). Introduction of an agent-based multiscale modular architecture for dynamic knowledge representation of acute inflammation. Theor. Biol. Med. Model. 5, 11. doi: 10.1186/1742-4682-5-11

Andremont, A., Marang, B., Tancrede, C., Baume, D., and Hill, C. (1989). Antibiotic treatment and intestinal colonization by Pseudomonas aeruginosa in cancer patients. Antimicrob. Agents Chemother. 33, 1400-1402. doi: 10.1128/AAC.33.8.1400

Apidianakis, Y., Pitsouli, C., Perrimon, N., and Rahme, L. (2009). Synergy between bacterial infection and genetic predisposition in intestinal dysplasia. Proc. Natl. Acad. Sci. U.S.A. 106, 20883-20888. doi: 10.1073/pnas.0911797106

Arthur, J. C., Perez-Chanona, E., Mühlbauer, M., Tomkovich, S., Uronis, J. M., Fan, T. J., et al. (2012). Intestinal inflammation targets cancerinducing activity of the microbiota. Science 338, 120-123. doi: 10.1126/science. 1224820

Babrowski, T., Romanowski, K., Fink, D., Kim, M., Gopalakrishnan, V., Zaborina, O., et al. (2013). The intestinal environment of surgical injury transforms Pseudomonas aeruginosa into a discrete hypervirulent morphotype capable of causing lethal peritonitis. Surgery 153, 36-43. doi: 10.1016/j.surg.2012.06.022

Bangi, E., Pitsouli, C., Rahme, L. G., Cagan, R., and Apidianakis, Y. (2012). Immune response to bacteria induces dissemination of ras-activated drosophila hindgut cells. EMBO Rep. 13, 569-576. doi: 10.1038/embor.2012.44

Berx, G., and van Roy, F. (2009). Involvement of members of the cadherin superfamily in cancer. Cold Spring Harb. Perspect. Biol. 1, a003129. doi: 10.1101/cshperspect.a003129

Bos, M. M., Smeets, L. S., Dumay, I., and de Jonge, E. (2013). Bloodstream infections in patients with or without cancer in a large community hospital. Infection 41, 949-988. doi: 10.1007/s15010-0130468-1

Breitschwerdt, E. B., Linder, K. L., Day, M. J., Maggi, R. G., Chomel, B. B., and Kempf, V. A. (2013). Koch's postulates and the pathogenesis of comparative infectious disease causation associated with bartonella species. J. Comp. Pathol. 148, 115-125. doi: $10.1016 /$ j.jcpa.2012.12.003
Cavallaro, U., and Christofori, G. (2004). Cell adhesion and signalling by cadherins and ig-CAMs in cancer. Nat. Rev. Cancer 4, 118-132. doi: $10.1038 / \mathrm{nrc1} 276$

Christofi, T., and Apidianakis, Y. (2013). Rasoncogenic drosophila hindgut but not midgut cells use an inflammation-like program to disseminate to distant sites. Gut Microbes 4, 54-59. doi: 10.4161/gmic. 22429

Collins, D., Hogan, A. M., and Winter, D. C. (2011). Microbial and viral pathogens in colorectal cancer. Lancet Oncol. 12, 504-512. doi: 10.1016/S14702045(10)70186-8

Craven, D. E., Kunches, L. M., Lichtenberg, D. A., Kollisch, N. R., Barry, M. A., Heeren, T. C., et al. (1988). Nosocomial infection and fatality in medical and surgical intensive care unit patients. Arch. Intern. Med. 148, 1161-1168. doi: 10.1001/archinte.1988.00380050165024

de Lima, C. D. M., Calegari-Silva, T. C., Pereira, R. M. S., de Oliveira Lima Santos, S. A., Lopes, U. G., Plotkowski, M. C. M., et al. (2012). ExoU activates NF- $\kappa \mathrm{B}$ and increases IL-8/KC secretion during Pseudomonas aeruginosa infection. PLoS ONE 7:e41772. doi: 10.1371/journal.pone. 0041772

Driscoll, J. A., Brody, S. L., and Kollef, M. H. (2007). The epidemiology, pathogenesis and treatment of Pseudomonas aeruginosa infections. Drugs 67, 351-368. doi: 10.2165/00003495-20076703000003

Dunn, G. P., Ikeda, H., Bruce, A. T., Koebel, C. Uppaluri, R., Bui, J., et al. (2005). Interferongamma and cancer immunoediting. Immunol. Res. 32, 231-245. doi: 10.1385/IR:32:1-3:231

Evan, G., and Littlewood, T. (1998). A matter of life and cell death. Science 281, 1317-1322. doi: $10.1126 /$ science.281.5381.1317

Gellatly, S. L., and Hancock, R. E. (2013). Pseudomonas aeruginosa: new insights into pathogenesis and host defenses. Pathog. Dis. 67, 159-173. doi: 10.1111/2049-632X. 12033

Hardy, R. G., Meltzer, S. J., and Jankowski, J. A. (2000). ABC of colorectal cancer. Molecular basis for risk factors. BMJ 321, 886-889. doi: 10.1136/bmj.321.7265.886

Hentges, D. J., Stein, A. J., Casey, S. W., and Que, J. U. (1985). Protective role of intestinal flora against infection with Pseudomonas aeruginosa in mice: influence of antibiotics on colonization resistance. Infect. Immun. 47, 118-122.

Holt, P. G., Strickland, D. H., Wikstrom, M. E., and Jahnsen, F. L. (2008). Regulation of immunological homeostasis in the respiratory tract. Nat. Rev. Immunol. 8, 142-152. doi: 10.1038/ nri2236

Hussain, S. P., Hofseth, L. J., and Harris, C. C. (2003). Radical causes of cancer. Nat. Rev. Cancer 3, 276-285. doi: 10.1038/ nrc1046

Kerr, K. G., and Snelling, A. M. (2009). Pseudomonas aeruginosa: a formidable and ever-present adversary. J. Hosp. Infect. 73, 338-344. doi: 10.1016/j.jhin.2009.04.020

Levison, M. E. (1973). Effect of colon flora and shortchain fatty acids on growth in vitro of Pseudomonas aeruginosa and enterobacteriaceae. Infect. Immun. $8,30-35$.
Long, J., Zaborina, O., Holbrook, C., Zaborin, A., and Alverdy, J. (2008). Depletion of intestinal phosphate after operative injury activates the virulence of $P$. aeruginosa causing lethal gut-derived sepsis. Surgery 144, 189-197. doi: 10.1016/j.surg.2008.03.045

Lowe, S. W., Cepero, E., and Evan, G. (2004). Intrinsic tumour suppression. Nature 432, 307-315. doi: $10.1038 /$ nature 03098

Macdonald, I. A., and Kuehn, M. J. (2013). Stress-induced outer membrane vesicle production by Pseudomonas aeruginosa. J. Bacteriol. 195, 2971-2981. doi: 10.1128/JB. 02267-12

Marshall, J. C., Christou, N. V., and Meakins, J. L. (1993). The gastrointestinal tract. The "undrained abscess" of multiple organ failure. Ann. Surg. 218, 111-119. doi: 10.1097/00000658-19930800000001

Montassier, E., Batard, E., Gastinne, T., Potel, G., and de La Cochetiere, M. F. (2013). Recent changes in bacteremia in patients with cancer: a systematic review of epidemiology and antibiotic resistance. Eur. J. Clin. Microbiol. Infect. Dis. 32, 841-850. doi: 10.1007/s10096-013-1819-7

Okuda, J., Hayashi, N., Okamoto, M., Sawada, S., Minagawa, S., Yano, Y., et al. (2010). Translocation of Pseudomonas aeruginosa from the intestinal tract is mediated by the binding of ExoS to an na, K-ATPase regulator, FXYD3. Infect. Immun. 78, 4511-4522. doi: 10.1128/IAI. 00428-10

Oliveira, A. L., de Souza, M., Carvalho-Dias, V. M., Ruiz, M. A., Silla, L., Tanaka, P. Y., et al. (2007). Epidemiology of bacteremia and factors associated with multi-drug-resistant gram-negative bacteremia in hematopoietic stem cell transplant recipients. Bone Marrow Transplant. 39, 775-781. doi: 10.1038/sj.bmt.1705677

Osmon, S., Ward, S., Fraser, V. J., and Kollef, M. H. (2004). Hospital mortality for patients with bacteremia due to staphylococcus aureus or Pseudomonas aeruginosa. Chest 125, 607-616. doi: 10.1378/chest.125.2.607

Papoff, P., Ceccarelli, G., d'Ettorre, G., Cerasaro, C., Caresta, E., Midulla, F., et al. (2012). Gut microbial translocation in critically ill children and effects of supplementation with pre- and pro biotics. Int. J. Microbiol. 2012, 151393. doi: 10.1155/2012/ 151393

Rafla, K., and Tredget, E. E. (2011). Infection control in the burn unit. Burns 37, 5-15. doi: 10.1016/j.burns.2009.06.198

Safdar, A., and Armstrong, D. (2001). Infectious morbidity in critically ill patients with cancer. Crit. Care Clin. 17, 531-570, vii-viii. doi: 10.1016/S0749-0704(05)70198-6

Sax, H., Uckay, I., Balmelli, C., Bernasconi, E. Boubaker, K., Muhlemann, K., et al. (2011). Overall burden of healthcare-associated infections among surgical patients. Results of a national study. Ann. Surg. 253, 365-370. doi: 10.1097/SLA.0b013e318202fda9

Schwitalla, S., Fingerle, A. A., Cammareri, P., Nebelsiek, T., Göktuna, S. I., Ziegler, P. K., et al. (2013). Intestinal tumorigenesis initiated by dedifferentiation and acquisition of stem-cell-like properties. Cell 152, 25-38. doi: 10.1016/j.cell.2012.12.012 
Seal, J. B., Alverdy, J. C., Zaborina, O., and An, G. (2011). Agent-based dynamic knowledge representation of Pseudomonas aeruginosa virulence activation in the stressed gut: towards characterizing host-pathogen interactions in gut-derived sepsis. Theor. Biol. Med. Model. 8, 33. doi: 10.1186/17424682-8-33

Selgrad, M., Malfertheiner, P., Fini, L., Goel, A., Boland, C. R., and Ricciardiello, L. (2008). The role of viral and bacterial pathogens in gastrointestinal cancer. J. Cell. Physiol. 216, 378-388. doi: $10.1002 /$ jcp. 21427

Stoodley, B. J., and Thom, B. T. (1970). Observations on the intestinal carriage of Pseudomonas aeruginosa. J. Med. Microbiol. 3, 367-375. doi: 10.1099/00222615-3-3-367

Stuart, B., Lin, J. H., and Mogayzel, P. J. Jr. (2010). Early eradication of Pseudomonas aeruginosa in patients with cystic fibrosis. Paediatr. Respir. Rev. 11, 177-184. doi: 10.1016/j.prrv.2010.05.003

Sundin, C., Thelaus, J., Broms, J. E., and Forsberg, A. (2004). Polarisation of type III translocation by Pseudomonas aeruginosa requires $\mathrm{PcrG}, \mathrm{PcrV}$ and PopN. Microb. Pathog. 37, 313-322. doi: 10.1016/j.micpath.2004.10.005

Tabrah, F. L. (2011). Koch's postulates, carnivorous cows, and tuberculosis today. Hawaii Med. J. 70, 144-148.

Tafe, L. J., and Ruoff, K. L. (2007). Streptococcus bovis: answers and questions. Clin. Microbiol. Newsl. 29, 49-55. doi: 10.1016/j.clinmicnews.2007.03.004

Thuong, M., Arvaniti, K., Ruimy, R., de la Salmoniere, P., Scanvic-Hameg, A., Lucet, J. C., et al. (2003).
Epidemiology of Pseudomonas aeruginosa and risk factors for carriage acquisition in an intensive care unit. J. Hosp. Infect. 53, 274-282. doi: 10.1053/jhin.2002.1370

Tjalsma, H., Boleij, A., Marchesi, J. R., and Dutilh, B. E. (2012). A bacterial driver - passenger model for colorectal cancer: beyond the usual suspects. Nat. Rev. Microbiol. 10, 575-582. doi: 10.1038/nrmicro2819

Toprak, N. U., Yagci, A., Gulluoglu, B. M., Akin, M. L., Demirkalem, P., Celenk, T., et al. (2006). A possible role of bacteroides fragilis enterotoxin in the aetiology of colorectal cancer. Clin. Microbiol. Infect. 12, 782-786. doi: 10.1111/j.1469-0691.2006. 01494.x

Vaupel, P., and Mayer, A. (2007). Hypoxia in cancer: significance and impact on clinical outcome. Cancer Metastasis Rev. 26, 225-239. doi: 10.1007/s10555-007-9055-1

Vikström, E., Bui, L., Konradsson, P., and Magnusson, K. E. (2009). The junctional integrity of epithelial cells is modulated by Pseudomonas aeruginosa quorum sensing molecule through phosphorylation-dependent mechanisms. Exp. Cell Res. 315, 313-326. doi: 10.1016/j.yexcr.2008. 10.044

Vuotto, F., Berthon, C., Lemaitre, N., Duhamel, A., Balkaran, S., Le Ray, E., et al. (2013). Risk factors, clinical features, and outcome of Pseudomonas aeruginosa bacteremia in patients with hematologic malignancies: a case-control study. Am. J. Infect. Control 41, 527-530. doi: 10.1016/j.ajic.2012.07.012
Worth, L. J., and Slavin, M. A. (2009). Bloodstream infections in haematology: risks and new challenges for prevention. Blood Rev. 23, 113-122. doi: 10.1016/j.blre.2008. 10.003

Zaborina, O., Kohler, J. E., Wang, Y., Bethel, C., Shevchenko, O., Wu, L., et al. (2006). Identification of multi-drug resistant Pseudomonas aeruginosa clinical isolates that are highly disruptive to the intestinal epithelial barrier. Ann. Clin. Microbiol. Antimicrob. 5, 14. doi: 10.1186/14760711-5-14

Received: 26 September 2013; accepted: 22 December 2013; published online: 07 January 2014.

Citation: Markou $P$ and Apidianakis $Y$ (2014) Pathogenesis of intestinal Pseudomonas aeruginosa infection in patients with cancer. Front. Cell. Infect. Microbiol. 3:115. doi: 10.3389/fcimb.2013.00115

This article was submitted to the journal Frontiers in Cellular and Infection Microbiology.

Copyright (C) 2014 Markou and Apidianakis. This is an open-access article distributed under the terms of the Creative Commons Attribution License (CC BY). The use, distribution or reproduction in other forums is permitted, provided the original author(s) or licensor are credited and that the original publication in this journal is cited, in accordance with accepted academic practice. No use, distribution or reproduction is permitted which does not comply with these terms. 